Nature Reviews Cancer | AOP, published online 12 April 2012; doi:10.1038/nrc3269

TUMOUR HETEROGENEITY

\title{
Darwin's finches
}

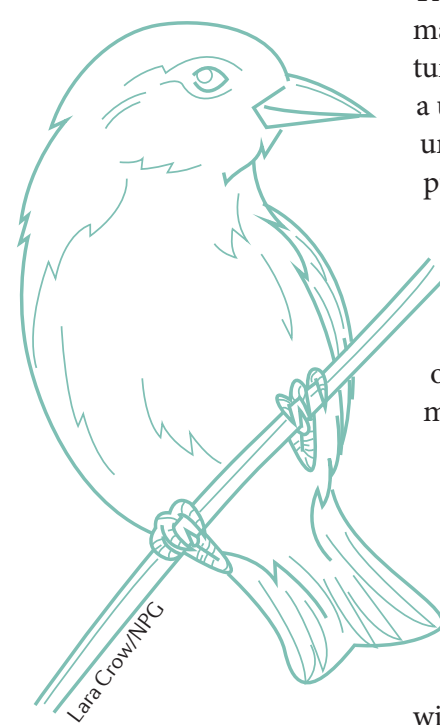

The era of personalized medicine makes much of the concept that a tumour that arises in a patient is a unique disease. Although this is undoubtedly true, a paper recently published in the New England

Journal of Medicine takes this one step further and clearly shows that, within a primary tumour, this uniqueness extends to regions of the tumour and its associated metastases.

Charles Swanton and colleagues examined the concept of intra-tumour heterogeneity (the idea that regions of a tumour are genetically different and so consist of subclones) in four patients who had been diagnosed with kidney cancer. Patient 1 had a clear-cell renal carcinoma with pulmonary and chest wall metastases. Initial sequencing revealed a $2 \mathrm{bp}$ inactivating mutation in the tumour suppressor gene von Hippel-Lindau (VHL; inactivation of $V H L$ is a characteristic of this disease). Wholeexome multiregion spatial sequencing of DNA was carried out on the pretreatment primary tumour biopsy and chest wall metastasis biopsy, and on nine regions of the tumour that were isolated from the excised kidney after treatment with the mTOR inhibitor everolimus, as well as a metastasis present in the perinephric fat and two regions of the excised chest wall metastasis. These were compared with germline DNA from the patient. Changes in chromosome number (ploidy) and chromosomal aberrations were also examined.

Of the driver genes known to be associated with clear-cell renal carcinoma, only the VHL mutation was ubiquitous, along with the loss of a region of $3 p$ that contained the second VHL allele and a SETD2 the disease

\section{complexity of}

allele. The methyltransferase SETD2 is another driver gene, and its other allele was mutated in three distinct ways in different areas of the tumour. These areas of the tumour showed reduced H3K36 trimethylation, indicating that this phenotype probably confers a selective advantage to tumour cells, so much so that individual subclones with different SETD2 mutations have survived within the tumour and metastases, supporting the concept of convergent evolution in tumours. Similarly, KDM5C, a histone demethylase, had the same missense and frameshift deletions in six regions of the tumour and a different splice site mutation in the metastases. mTOR was also found to be mutated in most regions of the primary tumour, except region 4 , which was the region most similar to the metastases. This is important because the mTOR inhibitor everolimus is a standard treatment for this disease, and these findings indicate that the different mTOR activity in the tumour could lead to different responses to this inhibitor. Perhaps most concerning was the finding that the different regions of the tumour and metastases segregated differently on the basis of known gene signatures of good and poor prognosis, suggesting that prognoses based on sampling one region of a primary tumour might not reflect the status of the entire tumour.

Analyses of tumours and metastases (when present) from three other patients with clear-cell renal carcinoma revealed similar findings, with

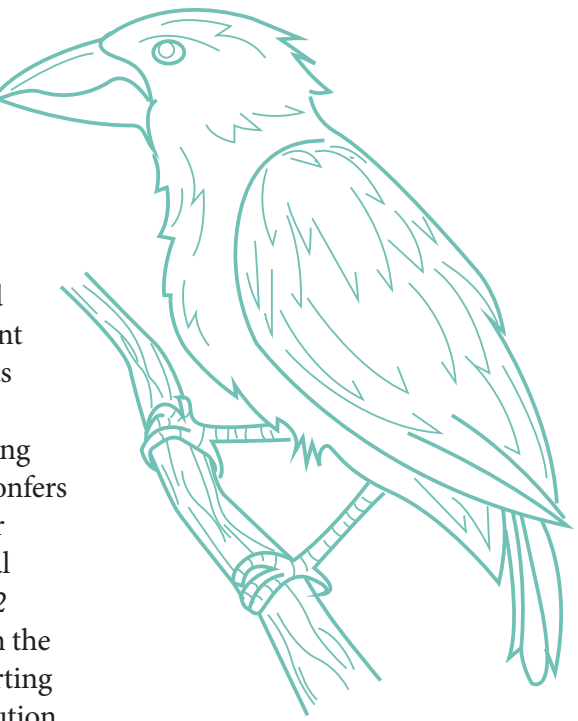

convergent evolution evident for SETD2 mutations in patient 2 and patient 4 , and PTEN mutations in patient 2. Chromosome $3 \mathrm{p}$ aberrations were the only consistent change across all four patient samples.

These data allow two important conclusions to be drawn: that the mutation of specific genes such as VHL and SETD2 are clearly crucial to the evolution of this disease and as such still represent robust therapeutic targets, and that genomic analyses based on a single tumour biopsy could potentially result in an incomplete picture of the complexity of the disease arising in an individual patient.

Nicola McCarthy

ORIGINAL RESEARCH PAPER Gerlinger, M. et al.

Intratumour heterogeneity and branched evolution revealed by multiregion sequencing. N. Engl.J. Med. 366, 883-892 (2012) 